Gut, 1987, 28, 835-843

\title{
Formation of a fibrin based gelatinous coat over repairing rat gastric epithelium after acute ethanol damage: interaction with adherent mucus
}

\author{
L A SEllers, A ALLEN, AND M K BENNETT
}

\begin{abstract}
From the Department of Physiological Sciences, Medical School, University, Newcastle upon Tyne and Department of Pathology, Freeman Road Hospital, Newcastle upon Tyne
\end{abstract}

SUMMARY A gelatinous coat, heterogeneous in appearance, was formed over damaged rat gastric mucosa recovering from acute ethanol injury. This coat, in places $1.6 \mathrm{~mm}$ thick (median thickness $680 \mu \mathrm{m}$ ), was 10 times thicker than the translucent layer of adherent mucus (median thickness $70 \mu \mathrm{m}$ ) covering the undamaged mucosa. Immunohistochemistry and periodic acid Schiff staining showed this gelatinous coat to be predominantly a fibrin gel with an exterior layer rich in mucus and necrotic cells. The plasma clotting time was significantly decreased in vitro by pig gastric mucus gel and soluble mucus glycoprotein ( $90 \%$ and $13 \%$ respectively) suggesting that in vivo the mucus layer remaining after epithelial damage could act as a template for fibrinogen-fibrin conversion. These results show that a fibrin based gelatinous coat, quite distinct from the adherent mucus layer and with considerable protective potential could be formed over the repairing rat gastric mucosa after acute ethanol damage.

A thin layer of water insoluble mucus gel can be observed adherent to the undamaged gastric mucosal surface: in man and rat the median thickness is about $180 \mu \mathrm{m}$ and $80 \mu \mathrm{m}$ respectively.' This adherent mucus layer provides the physical basis for the proposed mucus:bicarbonate barrier, a first line in mucosal defence against the endogenous aggressors, acid and pepsin..$^{--5}$ By forming a stable unstirred layer on the mucosa, mucus supports surface neutralisation of acid by epithelial bicarbonate. ${ }^{6}$ Pepsin cannot diffuse through the continuous mucus layer ${ }^{7}$ and consequently provides a protective barrier between the epithelium and pepsin in the lumen. Pepsinolysis will dissolve the adherent mucus layer at its luminal surface, the continuity of the barrier being maintained by fresh mucus secretion. ${ }^{*}$

The mucus:bicarbonate barrier is part of the mucosal protective mechanism that has evolved against the endogenous aggressors, acid and pepsin.

Address for correspondence: Professor A Allen. Dept of Physiological Sciences, Medical School. University of Newcastle upon Tyne, Framlington Place. Newcastle upon Tyne NE2 4HH.

Received for publication 7 November 1986.
It does not, however, appear initially to offer satisfactory protection to exogenous mucosal damaging agents, such as ethanol $(>40 \%)$, non-steroidal antiinflammatory drugs or hypertonic solutions - for example, $2 \mathrm{M} \mathrm{NaCl} .^{9}$ All these agents permeate the adherent mucus gel layer and when applied topically to the mucosal surface will produce extensive destruction of the epithelial cell layer and in many cases focal haemorrhagic lesions. ${ }^{1012}$ If mucosal damage is acute and confined to the epithelium with the basal lamina remaining intact, complete and rapid repair will occur through replacement of necrotic cells by preformed cells migrating from the gastric pits. ${ }^{12-15}$ This process of re-epithelialisation has been studied in vitro in frog and guinea pig as well as in vivo in rat and $\operatorname{man}^{16-19}$ and is often complete within one hour. With more severe damage, extending deeper into the mucosa beneath the epithelial layer, oedema, vascular congestion and haemorrhage as well as destruction of the pit cells and lamina propria will occur, thus delaying re-epithelialisation. After such damage with associated inflammation, healing will take days to complete. ${ }^{\text {is }}$ 
Histological studies after acute mucosal damage by ethanol have shown a massive release of gelatinous material, assigned primarily to mucus and necrotic cells, covering the newly re-epithelialising mucosa. ${ }^{1.215}$ A plasma exudate is associated with the formation of this coat and studies have also noted fibrin (from dimensions on electron micrographs) at the damaged mucosal surface. ${ }^{21-22}$ Such observations suggested to us that this thick, gelatinous coat might be of a different nature from the layer of translucent adherent mucus seen on undamaged or prostaglandin stimulated mucosa. ${ }^{1923}$ Here we report that after ethanol damage the nature of the adherent gastric mucus gel is dramatically changed because of an exudation of fibrinogen, resulting in a composite gel of fibrin, mucus and necrotic cells.

\section{Methods}

\section{ANIMALS}

Male Wistar rats (180-220 g) fasted for 48 hours were used. After pentobarbitone anaesthesia $(60 \mathrm{mg} / \mathrm{kg})$ the stomach was exposed and the oesophagus and duodenum ligated excluding major blood vessels and nerves. In all following procedures $70 \%(\mathrm{v} / \mathrm{v})$ ethanol, $0.9 \%(\mathrm{w} / \mathrm{v})$ sodium chloride or formal saline $(10 \%(\mathrm{v} / \mathrm{v})$ formalin in $0.9 \%(\mathrm{w} / \mathrm{v})$ sodium chloride) were injected into and withdrawn from the gastric lumen by a hypodermic needle inserted through the forestomach. The volume of each fluid injected into the stomach was $4 \mathrm{ml}$, which inflated the stomach to ensure uniform exposure of the mucosal surface to the solution. When $4 \mathrm{ml} 0.9 \%(\mathrm{w} / \mathrm{v})$ sodium chloride was incubated in the stomach for one hour the abdominal cavity was closed by suturing.

The experimental system of Lacy and Ito $^{14}$ was used to prepare the thick gelatinous coat formed after acute ethanol damage. A ligated stomach of an anaesthetised rat was filled with $70 \%(\mathrm{v} / \mathrm{v})$ ethanol for 45 seconds, a process that immediately destroys $96 \%$ of the superficial epithelium. ${ }^{1+}$ The mucosa was then exposed to $0.9 \%(\mathrm{w} / \mathrm{v})$ sodium chloride for one hour during which time over $90 \%$ has been shown to be re-epithelialised. The thickness of the adherent mucus layer or of the gelatinous coat formed after ethanol damage, was measured on unfixed sections by the method of Kerss et al. ${ }^{\prime}$ In other animals the stomach was fixed by an injection of formal saline, removed and preserved in the same solution. The gastric mucosa was routinely processed after formalin fixation in paraffin wax: alternate (vertical) sections (60 sections) were stained using either a peroxidase-antiperoxidase technique for fibrinogen, or periodic acid Schiff for mucin (PAS). The peroxidase-antiperoxidase technique ${ }^{2-}$ was as follows: after dewaxing the paraffin sections, the endogenous peroxidase activity was blocked using hydrogen peroxide with methanol and non-specific background staining was reduced by normal swine serum. The sections were then treated with polyvalent rabbit antihuman fibrinogen (Dakopatts, Copenhagen, diluted 1/200; at various times from five to 30 minutes). The antibody was visualised using rabbit antiperoxidase-peroxidase complex (PAP, diluted 1/50 for 30 minutes) and diaminobenzidine tetrahydrochloride. Finally the sections were counterstained, dehydrated, cleared in xylol and mounted in DPX.

Fasted rats were divided into the following experimental groups: group I: fasted rats; group II: saline control - anaesthetised rats with ligated stomachs filled with $0.9 \%(\mathrm{w} / \mathrm{v})$ sodium chloride for 45 seconds, followed by washing out and then incubation for one hour with $0.9 \%(\mathrm{w} / \mathrm{v})$ sodium chloride; group III: ethanol control - anaesthetised rats with ligated stomachs filled with $70 \%(\mathrm{v} / \mathrm{v})$ ethanol for 45 seconds; group IV: 're-epithelialised mucosa' - anaesthetised rats with ligated stomachs filled with $70 \%(\mathrm{v} / \mathrm{v})$ ethanol for 45 seconds followed by washing out and then incubation for one hour with $0.9 \%(w / v)$ sodium chloride.

The rate of fibrin formation from plasma was assayed as follows: plasma was obtained from the Regional Blood Transfusion Centre in citrated form: one part $3.8 \%(w / v)$ sodium citrate to nine parts plasma. Platelets were removed by centrifugation $(1000 \mathrm{~g} \times 10 \mathrm{~min})$ in plastic tubes. For measurement of coagulation time all solutions were previously equilibrated at $37^{\circ} \mathrm{C}$. Plasma $(0 \cdot 1 \mathrm{ml})$ was mixed by gentle tapping with the test agent $(0 \cdot 1 \mathrm{ml})$ and left for 30 seconds. Coagulation was initiated by addition to the mixture of $0.1 \mathrm{ml}$ of $0.025 \mathrm{M}$ calcium chloride (blown in through a pipette) and the time for the first threads of fibrin to appear was measured. Measurement of coagulation time for each test solution was repeated in independent experiments, by two workers, at least six times.

Gastric mucus gel was prepared by carefully scraping the surface of the stomachs of freshly slaughtered pigs $^{\mathrm{x}}$ and the mucus stored frozen until required for plasma coagulation tests. The mucus gel was also homogenised for one minute in four volumes of $0.9 \%$ $(\mathrm{w} / \mathrm{v})$ sodium chloride and centrifuged $(6000 \mathrm{~g} \times$ one hour) to prepare a solubilised glycoprotein preparation (supernatant).

Statistical analysis was by the Mann Whitney U test.

\section{Results}

Mucus thickness was measured by observation of unfixed $1.6 \mathrm{~mm}$ sections of mucosa (Table 1). The 
Table 1 Thickness of gelatinous layer over gastric epithelium: effect of acute ethanol damage

\begin{tabular}{|c|c|c|c|c|c|}
\hline \multirow[b]{2}{*}{ Group } & \multicolumn{4}{|c|}{$\begin{array}{l}\text { Thickness of gelatinous layer } \\
(\mu \mathrm{n})\end{array}$} & \multirow[b]{2}{*}{$\begin{array}{l}\text { Animals } \\
\text { (n) }\end{array}$} \\
\hline & Median & $\begin{array}{l}\text { Low } \\
\text { uppe }\end{array}$ & $\begin{array}{l}\text { rand } \\
\text { quartiles }\end{array}$ & Range & \\
\hline I Fasted & 80 & 60) & 110 & $10-250$ & 6 \\
\hline II Saline control & 70) & 50 & 100 & $10-220$ & 7 \\
\hline III Ethanol control & $20)^{*}$ & 10 & 40 & $0-130$ & 4 \\
\hline $\begin{array}{l}\text { IV 'Rc-epithelialised } \\
\text { mucosa' }\end{array}$ & $680^{*}$ & $5(0)$ & 890 & $40-1560$ & 7 \\
\hline
\end{tabular}

For each group of animals, three sections per stomach and at least 12 readings per section were taken. For details of treatment regime in respective groups sec Methods. *Significant difference from saline control $\mathrm{p}<0 .(0)$ by Mann Whitncy U-test.

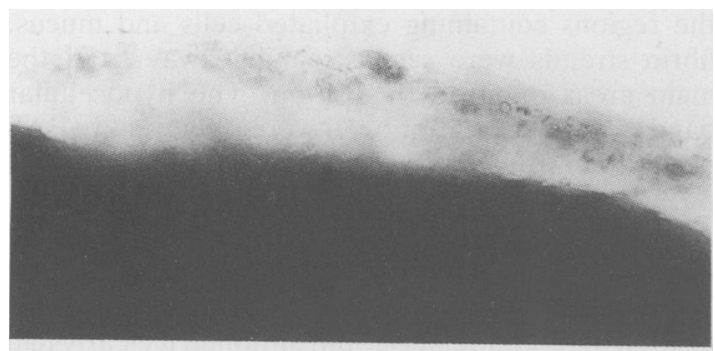

(a)

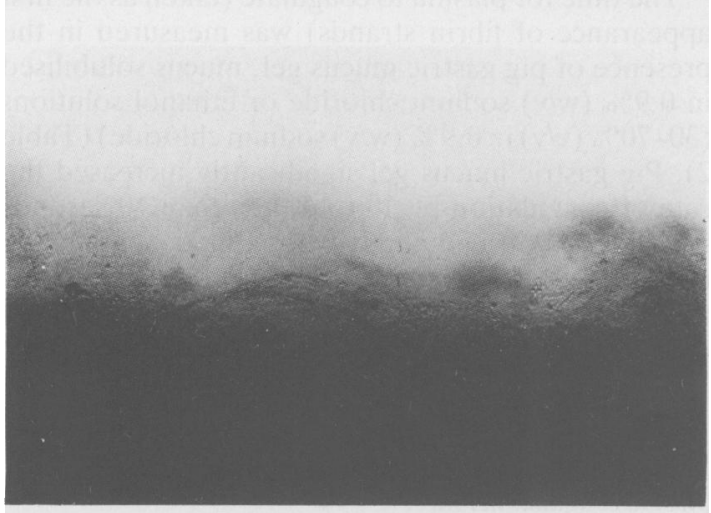

(b)

Fig. 1 (a) Saline control (group II): rat gastric mucosa exposed to $0.9 \%(w / v)$ sodium chloride for 45 seconds rapidly washed with the same solution followed by fresh $0.9 \%(w / v)$ sodium chloride for one hour. Unfixed sections (1.6 mm thick) viewed transversely under bright field.

(b) Ethanol control (group III): rat gastric mucosa exposed to $70 \%(v / v)$ ethanol for 45 seconds. Unfixed sections $(1.6$ mm thick) viewed transversely under bright field.

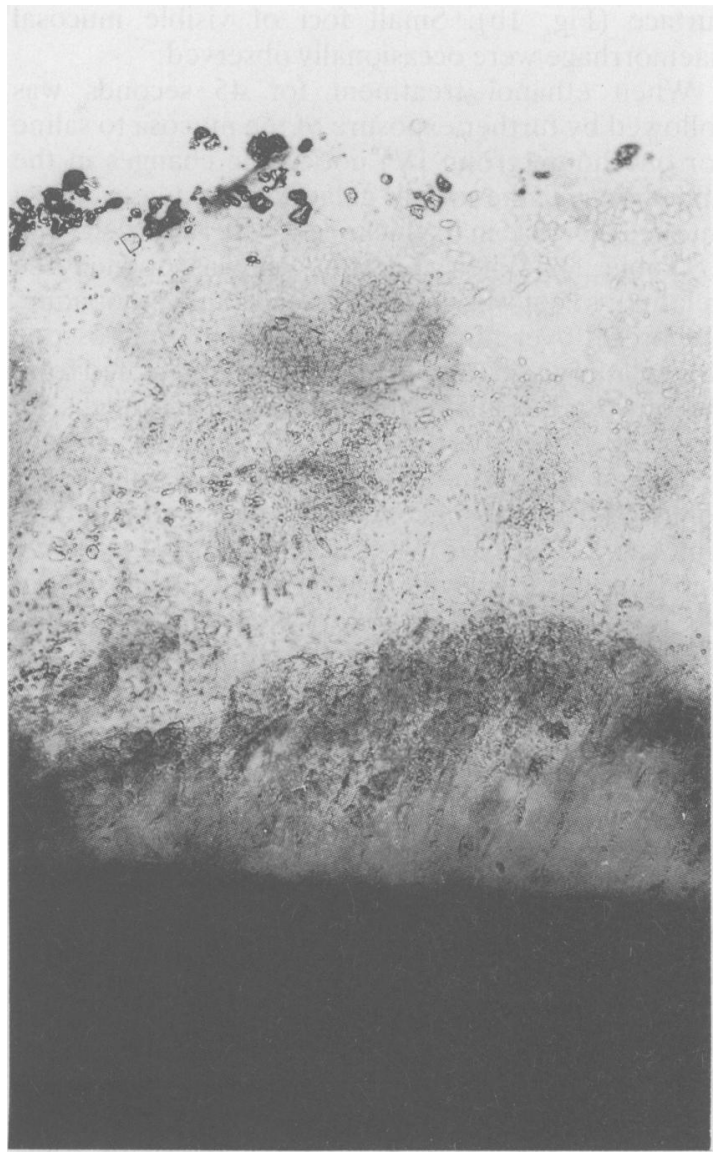

Fig. 2 'Re-epithelialised' mucosa (group IV): rat gastric mucosa exposed to $70 \%(\mathrm{v} / \mathrm{v})$ ethanol for 45 seconds rapidly washed with $0.9 \%(w / v)$ sodium chloride followed by $0.9 \%$ $(w / v)$ sodium chloride for one hour. Unfixed sections $(1 \cdot 6$ $\mathrm{mm}$ thick) viewed transversely under bright field.

adherent mucus layer covering the gastric mucosa of fasted rats (group I) was seen to be a thin, translucent layer of gel with a median thickness of $80 \mu \mathrm{m}$ (Fig. 1a) and minimum thickness $10 \mu \mathrm{m}$. After exposure to saline (group II) the median mucus thickness was not significantly different and the quality of gel unchanged from group I. The distribution of values in group II was, however, distinctly narrower than that for fasted animals. Although mucus thickness values over a single mucosa varied up to 10 -fold, median thickness values for each animal within a given treatment group were not significantly different from each other $(p<0 \cdot 05)$.

After exposure to ethanol for 45 seconds, median mucus thickness over the mucosa was significantly decreased to a median of $20 \mu \mathrm{m}$ (group III). In this group the mucus layer was very granular in appearance and not easily distinguishable from the mucosal 
surface (Fig. 1b). Small foci of visible mucosal haemorrhage were occasionally observed.

When ethanol treatment for 45 seconds was followed by further exposure of the mucosa to saline for one hour (group IV) noticeable changes in the appearance of the surface gelatinous coat, as well as a marked increase in its thickness, were observed (Fig. 2 , Table 1). When the stomach was opened the gelatinous coat was clearly visible as a thick, opaque, sloppy gel over the mucosa. Under the microscope this gelatinous coat could be readily demarcated from the mucosa but unlike the rigid adherent mucus gel layer seen on the undamaged mucosal surface (groups I and II), it could be cleanly and easily removed from the mucosa by physical manipulation. Thickness values for this gelatinous coat of up to 1.6 $\mathrm{mm}$ were observed with a median mucus thickness of $680 \mu \mathrm{m}$. In some instances, before measuring thickness, lumps of gel were seen in the lumen. These were not included in the thickness measurements which therefore were of minimum value. The adherent gelatinous coat was always continuous but values as thin as $40 \mu \mathrm{m}$ were occasionally recorded.

The distribution plot of mucus thickness values in $50 \mu \mathrm{m}$ intervals expressed as a percentage of the total number of readings is shown in Figure 3. These distributions emphasise the marked difference in thickness between the adherent mucus layer in the saline controls (group II) and the gelatinous coat over the re-epithelialised mucosa (group IV), where the distribution of thickness values was much wider.

Histological sections from saline control rats (group II) showed a normal mucosa with some

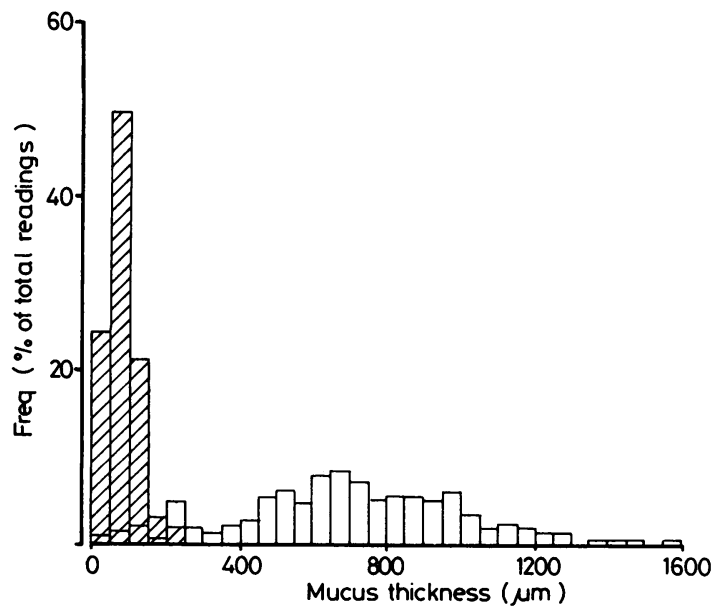

Fig. 3 Distribution profile of the frequency of thickness values in 50 um intervals for $(\square)$ adherent mucus on undamaged gastric mucosa from saline control rats (group II) and ( $\square$ ) gelatinous coat on repairing rat gastric mucosa after acute ethanol damage (group IV). fibrinogen staining in the mucosal blood vessels but none on the surface of the mucosa (Fig. 4). Within the surface epithelium cytoplasmic mucin (PAS) stained strongly, while only an occasional small blob of surface mucus was visible. Sections of mucosa taken after exposure to ethanol for 45 seconds (group III) showed the epithelium had lifted with submucosal oedema. Fibrinogen positive material was present in the superficial vessels of the lamina propria and in small amounts at the luminal surface (Fig. 5).

Marked differences were seen in the pattern of staining at the surface of the mucosa after exposure to ethanol for 45 seconds and isotonic saline for one hour (group IV). Thick bands of fibrinogen positive material were present in the gelatinous coat indicating fibrin deposition (Fig. 6). These fibrin containing areas were hypocellular while large numbers of exfoliated, pyknotic cells and PAS positive mucus were found above, proximal to the lumen (Fig. 7). Occasionally whole sheets of desquamated, necrotic epithelium were observed above the fibrin layer. In the regions containing exfoliated cells and mucus, fibrin strands were seen streaking away from the main areas of fibrin deposition. The hypocellular bands staining for fibrinogen were only weakly PAS positive. Fibrinogen was also observed in the lamina propria and within the congested blood vessels. Areas of regenerated epithelium were observed although re-epithelialisation was not complete. The peroxidase staining used here does not enable quantification of re-epithelialisation, although it clearly had occurred (Figs 6 and 7).

The time for plasma to coagulate (taken as the first appearance of fibrin strands) was measured in the presence of pig gastric mucus gel, mucus solubilised in $0.9 \%(\mathrm{w} / \mathrm{v})$ sodium chloride or ethanol solutions $(30-70 \%(\mathrm{v} / \mathrm{v})$ in $0.9 \%(\mathrm{w} / \mathrm{v})$ sodium chloride) (Table 2). Pig gastric mucus gel significantly increased the rate of coagulation by 10 -fold, less than 10 seconds for coagulation compared with 100 seconds in the control. Two similar experiments using mucus scraped from the surface of rat stomachs also decreased the plasma coagulation time by over a factor

Table 2 Effect of mucus gel, soluble mucus and ethanol on plasma coagulation time

\begin{tabular}{|c|c|c|c|}
\hline Agent & $n$ & $\begin{array}{l}\text { Coagulation time (sec) } \\
\text { Mean } \pm S E M\end{array}$ & $p$ \\
\hline $0.9 \%(w / v)$ sodium chloridc & 16 & $1(x) \pm 0.82$ & \\
\hline Pig gastric mucus gel & 6 & $7 \pm 0.67$ & ()$\cdot()() 1$ \\
\hline Soluble pig gastric mucus & 16 & $87 \pm 1 \cdot 8.5$ & $0 \cdot()(0) 1$ \\
\hline Rat gastric mucus gel & 2 & $5 \pm 3 \cdot(0)$ & \\
\hline Ethanol solution $30 \%(\mathrm{v} / \mathrm{v})$ & 6 & $3(1) 8 \pm 15 \cdot 8()$ & ()$\cdot()() 1$ \\
\hline
\end{tabular}

Significant difference from saline control by Mann Whitncy U-test. 


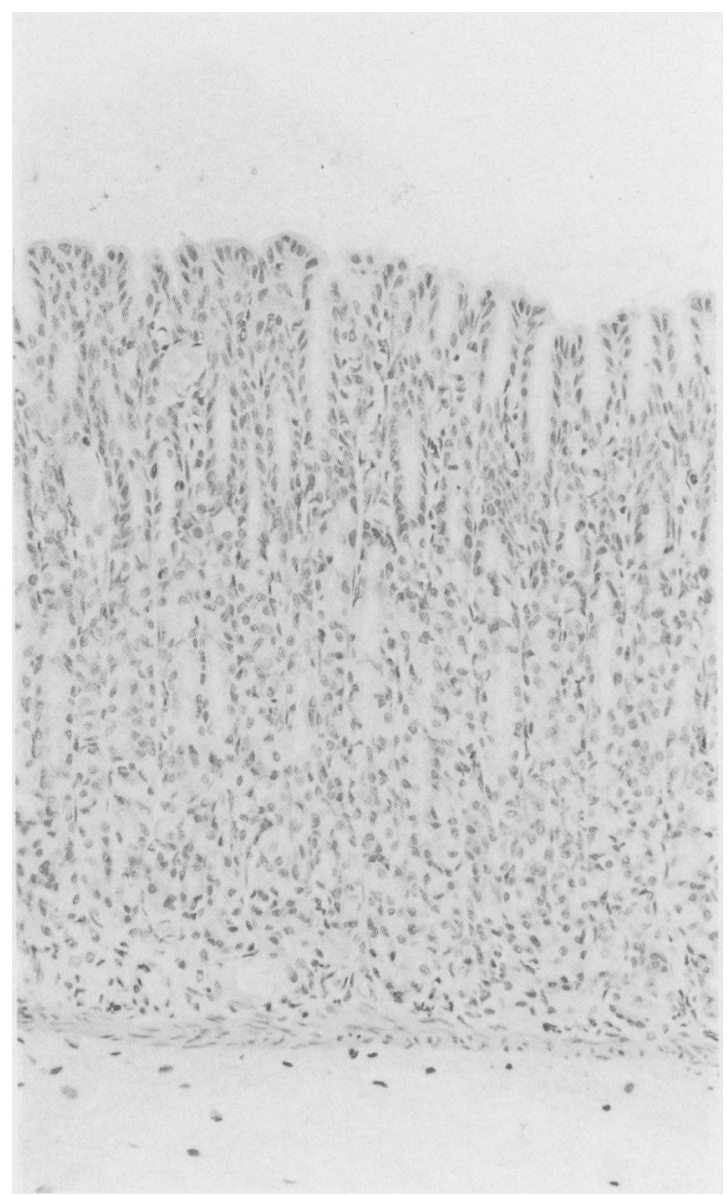

Fig. 4 Saline control (group II): rat gastric mucosa following exposure to $0.9 \%(w / v)$ sodium chloride for 45 seconds rapidly washed with the same solution followed by $0.9 \%(w / v)$ sodium chloride for one hour. The mucosa was formalin fixed and embedded in paraffin wax with sections stained by peroxidase-antiperoxidase for fibrinogen (counter stained with haematoxylin).

of 10 with respect to control values. The small quantities of mucus obtainable from rat stomachs compared to that from pig, however, made it necessary to use the latter for quantitative studies. The composit mucus/plasma gel formed in this in vitro coagulation was sloppy and somewhat opaque in appearance. Pig gastric mucus gel solubilised in (0.9\% (w/v) sodium chloride also significantly increased the rate of coagulation by $13 \%$. In higher concentrations of ethanol, $40 \%(\mathrm{v} / \mathrm{v})$ or above, coagulation did not occur for 15 minutes, while $30 \%(\mathrm{v} / \mathrm{v})$ ethanol significantly decreased the rate of coagulation by 3 fold.

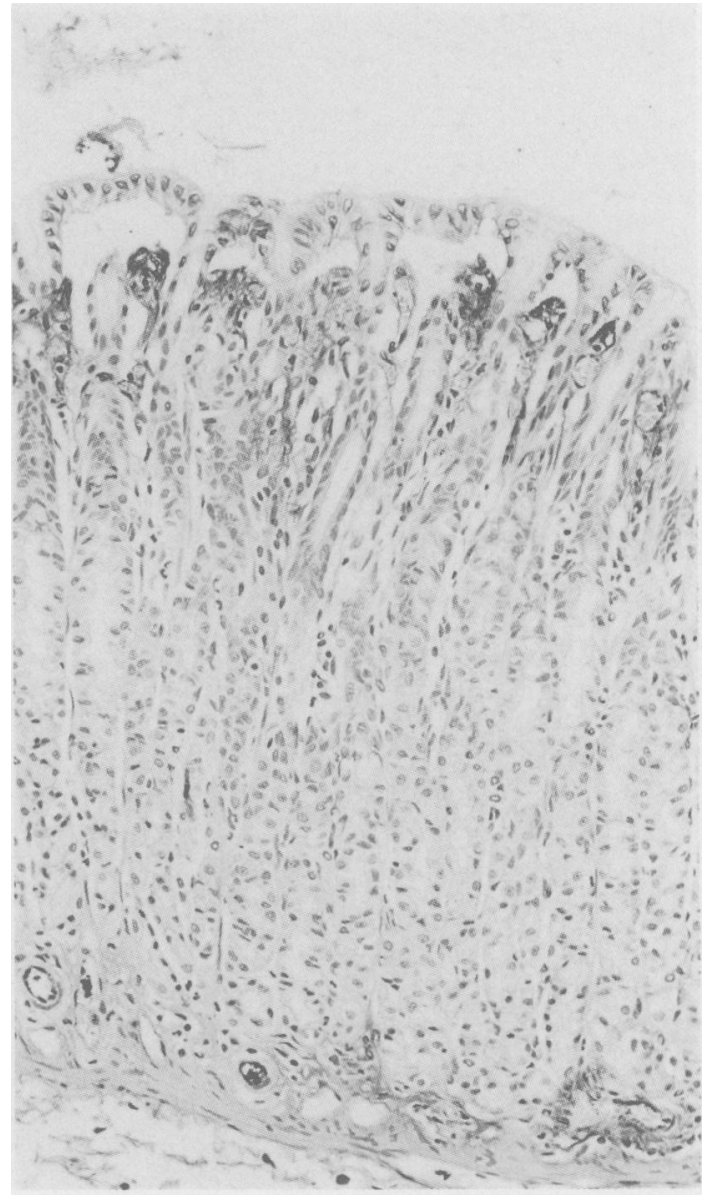

Fig. 5 Ethanol control (group III): gastric mucosa following exposure to $70 \%(\mathrm{v} / \mathrm{v})$ ethanol for 45 seconds. The mucosa, formalin fixed, was embedded in paraffin wax and sections stained with peroxidase-antiperoxidase for fibrinogen (counter stained with haematoxylin).

\section{Discussion}

Mucus adherent to unfixed sections from undamaged stomachs was observed as a translucent layer of gel clearly demarcated from the mucosa and bathing solution (Fig. 1a). The median thickness of the gastric mucus layer in fasted animals (group I) was 80 $\mu \mathrm{m}$ and was not significantly changed by exposure to isotonic sodium chloride for one hour; median thickness $70 \mu \mathrm{m}$, group II. These results are in agreement with previous studies using this method. "Is In this and many other studies (at least eight separate studies - approximately 1000 observations) on fasted 


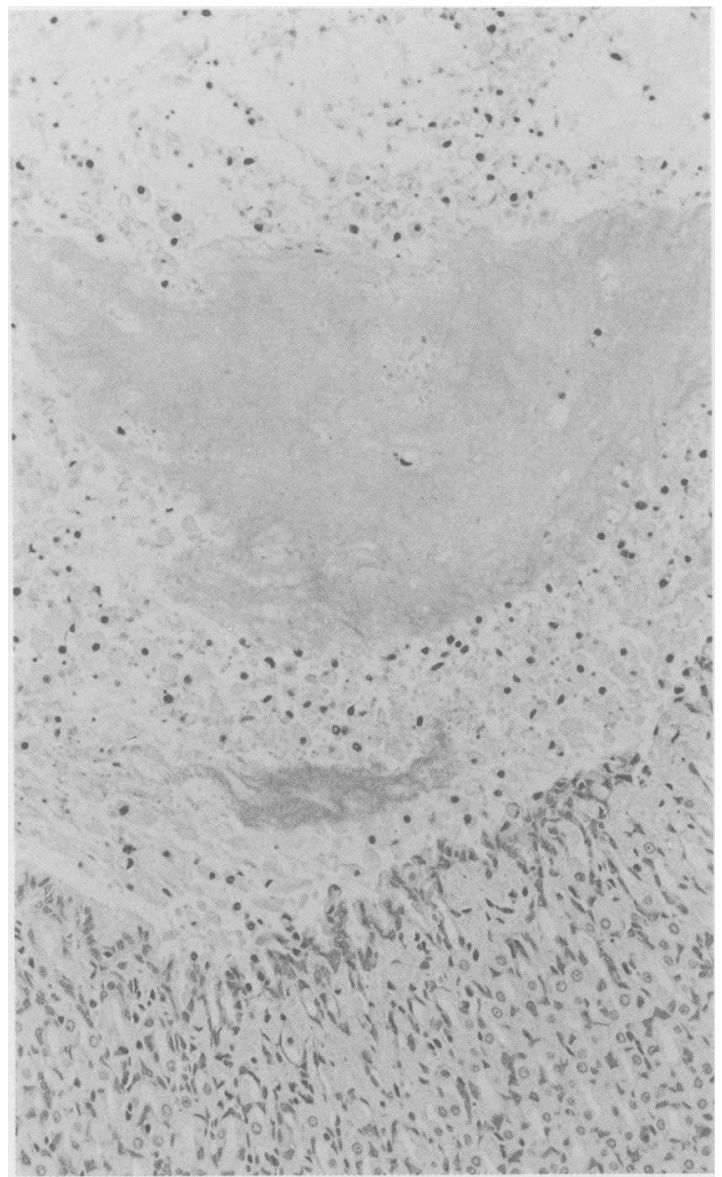

Fig. 6 'Re-epithelialised' mucosa (group IV): rat gastric mucosa following exposure to $70 \%(v / v)$ ethanol for 45 seconds rapidly washed with $0.9 \%(w / v)$ sodium chloride followed by $0.9 \%(w / v)$ sodium chloride for one hour. The mucosa, formalin fixed, was embedded in paraffin wax and sections stained with peroxidase-antiperoxidase for fibrinogen (counter stained with haematoxylin).

rats and those infused with saline, the mucus layer on unfixed gastric sections has always been observed to be continuous. Similarly in man the adherent gastric mucus layer is always seen to be continuous with a minimum thickness of $50 \mu \mathrm{m}$. The continuity of the mucus layer over undamaged mucosa has been questioned, however, because discontinuities have been observed on histologically fixed sections of rat gastric mucosa. ${ }^{21020}$ We consider such discontinuities to result from the fixation procedure as studies with mucus gel in situ and in vitro show it is distorted and dehydrated by histological fixatives - for example, alcohol and glutaraldehyde. ${ }^{27}$ Thus $5 \%(\mathrm{v} / \mathrm{v})$ glutaraldehyde has been shown to cause discontinuities

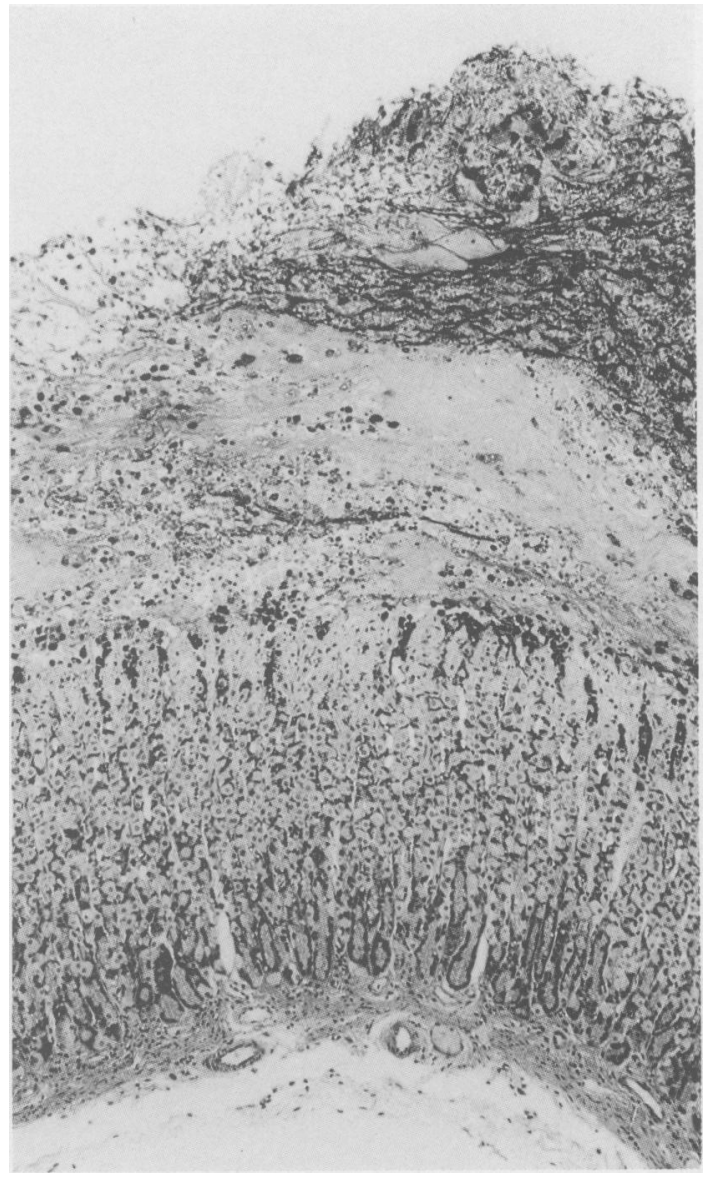

Fig. 7 'Re-epithelialised' mucosa (group IV): rat gastric mucosa following exposure to $70 \%(v / v)$ ethanol for 45 seconds rapidly washed with $0.9 \%(w / v)$ sodium chloride followed by $0.9 \%(w / v)$ sodium chloride for one hour. The mucosa, formalin fixed, was embedded in paraffin wax and sections stained with periodic acid Schiff for mucin.

and an overall shrinkage (of $45 \%$ ) in the thickness of the mucus layer on unfixed sections of mucosa to give values comparable with those seen for histologically fixed sections processed similarly. Further evidence for the detrimental effect of fixatives on the integrity of extracellular adherent mucus is apparent from its virtual absence on routinely fixed histological sections of undamaged gastric mucosa, although it is clearly visible on the mucosal surface before processing. In this study for example, no adherent mucus was observed on the gastric mucosa from starved animals after fixation and PAS staining (Fig. 4) although it was clearly present on unfixed sections (Fig. 1a). The particular effect of ethanol on adherent gastric mucus 
was shown in this study (on otherwise unfixed mucosa) by the marked reduction in mucus thickness to a median of $20 \mu \mathrm{m}$ with apparent discontinuities, following exposure to $70 \%(\mathrm{v} / \mathrm{v})$ ethanol for 45 seconds (group III, Fig. 1b and Table 1). In this treatment group the mucus layer was very granular in appearance and not easily differentiated from the mucosa. From the above evidence, it is considered that a continuous layer of mucus represents its true state in vivo. Further evidence for a continuous mucus layer comes from indirect measurements of mucus thickness using a slit lamp and pachymeter ${ }^{29}$ and the measurement of $\mathrm{pH}$ gradients at the mucosal surface. ${ }^{53131}$ In both these experimental systems discontinuities in the mucus layer were not seen.

The copious amounts of thick, opaque, gelatinous coat formed over the repairing mucosa after acute ethanol damage (group IV) was markedly different from the glistening, adherent mucus layer of the normal mucosa. The gelatinous coat was very granular and heterogeneous in appearance because of exfoliated cells. The median thickness of this gelatinous coat $(680 \mu \mathrm{m})$ was approaching a value of 10 times that of the median thickness of adherent mucus gel on undamaged mucosa. This increased thickness is all the more impressive when considered against the increase in adherent mucus thickness (measured by the same method) to a median of about $200 \mu \mathrm{m}$ after maximal stimulation by prostaglandin or carbachol to rats in vivo. ${ }^{14}$ These excessive amounts of surface gel associated with the repairing gastric mucosa suggested there was another gel forming component other than mucus glycoprotein. This component was shown by immunocytochemical studies to be fibrin.

The thick bands of staining for fibrinogen, present in the gelatinous coat covering the repairing mucosa indicated fibrin deposition. Exfoliated cells were found above and around the fibrin bands (only weakly PAS positive), admixed with material that was strongly PAS positive. It was not possible to determine whether smaller amounts of fibrin were present in the necrotic cell/mucus mass or whether some mucus was present in the fibrin layer. Sections from control rats - that is, no ethanol - showed no fibrinogen at the surface of the mucosa. Sections of mucosa taken after exposure to ethanol alone (45 seconds without the subsequent recovery period in sodium chloride) had mucosal surface oedema with some fibrinogen positive material in the lamina propria and traces at the luminal surface. These observations showed that over the repairing mucosa the thin, translucent layer of adherent mucus gel had been replaced by a 10 -fold thicker gelatinous coat consisting of fibrin associated with a largely exterior mass of necrotic cells and mucus.
Measurement of plasma coagulation time in vitro suggested that mucus remaining after mucosal damage may have a role as a template for the formulation of the fibrin gel in vivo. The rate of plasma coagulation increased 10 -fold in the presence of mucus gel and significantly increased in the presence of soluble mucus (Table 2). The insoluble mucus might be expected to facilitate the fibrinogen: fibrin conversion by virtue of its surface but the effect of soluble mucus suggests there may be more specific interactions between mucus glycoprotein and components of the clotting cascade. It is interesting in this context that an association between mucin from adenocarcinomas and fibrin occurs in cases of venous thrombosis and non-bacterial thrombotic endocardities. ${ }^{32}{ }^{33} \mathrm{~A}$ fibrin gel has also been shown to form in the damaged gall bladder after lysolethicin perfusion. ${ }^{3 .}$

Formation of this fibrin gelatinous coat would be expected to provide effective protection of reepithelialising cells from the endogenous aggressors acid and pepsin in the lumen. This protection would be further augmented by an essentially unlimited neutralising capacity from the plasma exudate. Recent studies show that this gelatinous coat protects re-epithelialisation against $0.05 \mathrm{M} \mathrm{HCl}$ in the luminal solution. ${ }^{35}$ The gelatinous coat also appears to have protective properties against ethanol, not shown by the adherent mucus layer. Thus in contrast with the original adherent mucus barrier it has been shown to protect the newly regenerated epithelium from further mucosal damage on re-exposure to $70 \%(\mathrm{v} / \mathrm{v})$ ethanol. ${ }^{21}=2$ If the gelatinous coat is removed from the surface the epithelium is as sensitive to ethanol damage, if not more so, than the original epithelium. The retention on histologically fixed sections of the gelatinous coat but not adherent mucus, is evidence for the resistance to ethanol of the former but not the latter (compare Fig. 4 with Figs 6 and 7).

It is evident that after acute ethanol damage the rat gastric mucosa is protected against further mucosal damage by a substantial gelatinous coat of fibrin with mucus and necrotic cells. For formation of this gelatinous-fibrin coat plasma exudation is necessary. ${ }^{22}$ It remains to be seen whether such protective coats are formed in other situations of mucosal repair after acute damage in animal models and man. Where such a fibrin based gelatinous coat is formed at the mucosal surface it would be expected to be a major contributor to maintaining mucosal protection.

These studies show that exudates of gel after mucosal damage can in large part be fibrin rather than mucus glycoproteins. There are substantial differences in properties and appearance between the adherent mucus layer characteristic of the undamaged mucosa and the fibrin based gel formed 
over repairing damaged mucosa. Such differences between the two types of surface gel put in perspective the conflicting views on the nature of the mucus layer between those working primarily with the ethanol damaged model and those working with unfixed sections of undamaged mucosa.

We would like to thank $\mathrm{Mr} \mathrm{N} \mathrm{J} \mathrm{H} \mathrm{Carroll} \mathrm{and} \mathrm{Mr} \mathrm{C}$ Hudson for technical help.

\section{References}

1 Kerss S, Allen A, Garner A. A simple method for measuring thickness of the mucus gel layer adherent to rat, frog and human gastric mucosa: Influence of feeding, prostaglandin, $\mathrm{N}$-acetylcysteine and other agents. Clin Sci 1982; 63: 187-95.

2 Hollander F. The two-component mucous barrier. Arch Intern Med 1954; 94: 107-20.

3 Heatley NG. Mucosubstance as a barrier to diffusion. Gastroenterology 1959; 37: 313-7.

4 Allen A, Garner A. Gastric mucus and bicarbonate secretion and their possible role in mucosal protection. Gut 1980; 21: 249-62.

5 Williams SE, Turnberg LA. Studies of the 'protective' properties of gastric mucus: evidence for a mucus bicarbonate barrier. Gut 1981; 22: 94-6.

6 Flemström G, Garner A. Gastroduodenal $\mathrm{HCO}_{3}$-transport: characteristics and proposed role in acidity regulation and mucosal protection. Am J Physiol 1982; 242: G183-93.

7 Allen A. The structure and function of gastrointestinal mucus. In: Harmon JW, ed. Basic mechanisms of gastrointestinal mucosal injury and protection. Baltimore: Williams \& Wilkins, 1981: 351-67.

8 Bell AE, Sellers LA, Allen A, Cunliffe WJ, Morris ER, Ross-Murphy SB. Properties of gastric and duodenal mucus: effect of proteolysis, disulphide reduction, bile, acid, ethanol and hypertonicity on mucus gel structure. Gastroenterology 1985; 88: 269-80.

9 McQueen S, Hutton DA, Allen A, Garner A. Gastric and duodenal surface mucus gel thickness in rat: Effect of prostaglandins and damaging agents. Am J Physiol 1983; 8: 388-94.

10 Robert A. Cytoprotection by prostaglandins. Gastroenterology 1979; 77: 761-7.

11 Robert A, Nezamis JE, Lancaster C, Hanchar AJ. Cytoprotection by prostaglandins in rats. Prevention of gastric necrosis produced by alcohol, $\mathrm{HCl}, \mathrm{NaOH}$, hypertonic $\mathrm{NaCl}$ and thermal injury. Gastroenterology 1979; 77: 433-43.

12 Lacy ER, Ito S. Microscopic analysis of ethanol damage to rat gastric mucosa after treatment with a prostaglandin. Gastroenterology 1982; 82: 619-25.

13 Morris GP, Wallace JL. The roles of ethanol and acid in the production of gastric mucosal erosion in rats. Virchows Arch /Cell Pathol] 1981; 38: 23-38.

14 Lacy ER, Ito S. Rapid epithelial restitution of the rat gastric mucosa after ethanol injury. Lab Invest 1984; 51: $573-83$.
15 Silen W. Ito S. Mechanisms for rapid re-epithelialisation of the gastric mucosal surface. Ann Rev Physiol 1985; 47: $217-9$

16 Baskin WN, Ivey KJ, Krause WJ, Jeffrey GE, Gemmell RT. Aspirin induced ultrastructural changes in human gastric mucosa. Correlation with potential differences. Ann Intern Med 1976; 85: 299-303.

17 Lacy ER, Ito $S$. Ethanol induced insult to the superficial rat gastric epithelium: a study of damage and rapid repair. In: Allen A, et al, eds. Mechanisms of mucosal protection in the upper gastrointestinal tract. New York: Raven Press, 1984: 49-55.

18 Rutten MJ, Ito S. Luminal acid effects on reconstitution of damaged guinea pig mucosa in vivo. In: Allen A, et al, eds. Mechanisms of mucosal protection in the upper gastrointestinal tract. New York: Raven Press, 1984: 41-7.

19 Svanes K, Ito S, Takeuchi K, Silen W. Restitution of the surface epithelium of the in vitro frog gastric mucosa after damage with hyperosomolar sodium chloride morphologic and physiologic characteristics. Gastroenterology 1982; 82: 1409-26.

20 Morris GP, Harding PL, Wallace JL. A functional model for extracellular gastric mucus in the rat. Virchows Arch /Cell Pathol] 1984; 46: 239-51.

21 Ito S, Lacy ER. Morphology of rat gastric mucosal damage, defense, and restitution in the presence of luminal ethanol. Gastroenterology 1985; 88: 250-60.

22 Lacy ER. Gastric mucosal resistance to a repeated ethanol insult. Scand J Gastroenterol 1985; 20: 250-60.

23 Sellers LA, Allen A, Bennett MK, Hudson C. Fibrinmucus interactions at the gastric mucosal surface and their role in mucosal protection. [Abstract]. Gut 1986; 27: A593-4.

24 Burns J. Immunocytochemical methods and their application in routine Laboratory. In: Antony PP, Wolf A, eds. Recent advance in histopathology. No. 10. Edinburgh: Churchill Livingstone, 1978: 337-50.

25 Sellers LA, Carroll NJH, Allen A. Misoprostol induced increases in adherent gastric mucus thickness and luminal mucus output. Dig Dis Sci 1986; 31: 91S-5S.

26 Morris GP. The myth of the mucus barrier. Gastroenterol Clin Biol 1984; 9: 106-7.

27 McQueen S, Allen A, Garner A. Measurement of gastric and duodenal mucus gel thickness. In: Allen A.et al, eds. Mechanisms of mucosal protection in the upper gastrointestinal tract. New York: Raven Press, 1984: $215-21$.

28 Allen A, Hutton DA, Leonard AJ, Pearson JP. Sellers LA. The role of mucus in the protection of the gastroduodenal mucosa. Scand J Gastroenterol 1986; 21: suppl. 125: 71-7.

29 Bickel M, Kauffman GL. Gastric mucus gel thickness: effects of distension, 16,16-dimethyl prostaglandin E2 and carbenoxolone. Gastroenterology 1981: 80: 77()-5.

30) Takeuchi KD, Magee D, Critchlow J, Silen W. Role of $\mathrm{pH}$ gradient of mucus in protection of gastric mucosa. Gastroenterology 1983; 84: 331-40.

31 Flemström G, Kivilaakso E. Demonstration of a $\mathrm{pH}$ gradient at the luminal surface of rat duodenum in vivo and its dependence on mucosal alkaline secretion. Gastroenterology 1983; 84: 787-94.

32 Brain MC, Azzopardi JG. Baker L. et al. Microangio- 
pathic haemolytic anaemia and mucin forming endocarcinoma. Br J Haem 1981; 18: 183-93.

33 Min K, Gyorkey F, Sato C. Mucin producing endenocarcinomas and non-bacterial thrombotic endocarditis. Cancer 1980; 45: 2374-82.

34 Neiderhiser DH. Fibrinogen secretion by the gall bladder: a potential role in gallstone formation. Clin Res 1983; 31: 765 .

35 Wallace JL, Whittle BJR. Role of mucus in the repair of gastric epithelial damage in the rat. Inhibition of epithelial recovery by mucolytic agents. Gastroenterology 1986; 91: 603-11. 